versus $4 \% ; p=0.03$ ), and lower prevalence of Raynaud's phenomenon (65\% versus $82 \%, p=0.002)$. Regarding cumulative manifestations, myositis ( $51 \%$ versus $15 \% ; p<0.001$ ), arthritis ( $43 \%$ versus $22 \% ; p=0.001$ ), and interstitial lung disease (ILD) $(60 \%$ versus $45 \%, \mathrm{p}=0.014)$ were more prevalent in patients with anti-PM/ $\mathrm{Scl}$ antibodies. In fact, those patients with anti- $\mathrm{Pm} / \mathrm{Scl}$ antibodies presented with FVC $(77.4 \% \pm 23.1 \%$ versus $85.8 \% \pm 23,1 \% ; p=0.006)$ and more severe ILD defined as FVC $<70 \%$ (41\% versus $24 \%$; $p=0.004)$. Death rate was similar in patients with and without $\mathrm{PM} / \mathrm{Scl}$ antibodies (18\% versus $17 \% ; \mathrm{p}=0.871)$. We did not find differences in terms of death rate nor in the causes of death (SSC and non-SSc related) according to the anti-PM/Scl antibodies profile. The 5- and 10 -years survival rates of patients with anti-PM/Scl antibodies were $91 \%$ and $82 \%$ respectively, without differences with those without these antibodies (93\% and $85 \%$, respectively)

Conclusion: In Spanish SSc patients, the presence of anti-PM/Scl antibodies confer a distinctive clinical profile. However, anti-PM/Scl antibodies do not play a role in the prognosis of these patients.

References:

[1] Stochmal A, Czuwara J, Trojanowska M, Rudnicka L. Antinuclear antibodies in systemic sclerosis: an update. Clin Rev Allergy Immunol 2020;58(1):40-51. doi: 10.1007/s12016-018-8718-8.

Acknowledgments: We gratefully acknowledge all investigators who are part of the RESCLE Registry. We also thank the RESCLE Registry Coordinating Centre, S\&H Medical Science Service, for their quality control data, logistic and administrative support and Prof. Salvador Ortiz, Universidad Autónoma de Madrid and Statistical Advisor S\&H Medical Science Service for the statistical analysis of the data presented in this paper.

Disclosure of InterestsNerea Iniesta-Arandia: None declared, Gerard Espinosa Speakers bureau: Glaxo-Smith-Kline, Janssen, Boehringer, Rovi, Alfredo Guillen del Castillo: None declared, Carles Tolosa Consultant of: Actelion pharmaceuticals, GSK, MSD., Gema Maria Lledó: None declared, Dolores Colunga Argüelles Consultant of: Actelion pharmaceuticals, GSK, MSD., Cristina GonzálezEchávarri: None declared, Luis Sáez-Comet: None declared, Norberto Ortego: None declared, Jose Antonio Vargas-Hitos: None declared, Manuel Rubio-Rivas: None declared, Mayka Freire: None declared, Juan José Rios: None declared, Monica Rodriguez-Carballeira: None declared, Luis Trapiella Martínez: None declared, Vicent Fonollosa Pla Speakers bureau: Actelion, Carmen Pilar Simeón-Aznar Consultant of: Actelion pharmaceuticals, GSK, MSD., on behalf of RESCLE Investigators, Autoimmune Diseases Study Group (GEAS): None declared

DOI: 10.1136/annrheumdis-2020-eular.3304

\section{SAT0317 HDL-CHOLESTEROL EFFLUX CAPACITY IS DOWNREGULATED IN PATIENTS WITH SYSTEMIC SCLEROSIS.}

1. Ferraz-Amaro ${ }^{1}$, D. F. Esmeralda ${ }^{1}$, V. Hernández-Hernández ${ }^{1}$, H. SánchezPérez $^{1}$, L. De Armas-Rillo² ${ }^{2}$ E. Armas González ${ }^{3}$, J. D. Machado ${ }^{4}$, F. DíazGonzález ${ }^{1}$. Division of Rheumatology, Hospital Universitario de Canarias, Tenerife, Spain, Santa Cruz de Tenerife, Spain; ${ }^{2}$ Universidad Europea de Canarias, Santa Cruz de Tenerife, Spain; ${ }^{3}$ Universidad de La Laguna, Departamento de Bioqui ímica, Microbiología, Biología Celular y Genética, La Laguna, Spain; ${ }^{4}$ Universidad de La Laguna, Santa Cruz de Tenerife, Spain

Background: It is well established that patients with systemic sclerosis (SS) show a disrupted lipid profile and an increased cardiovascular risk. Cholesterol efflux capacity (CEC) is the ability of high-density lipoprotein (HDL)-cholesterol to accept cholesterol from macrophages. CEC has been linked to cardiovascular events in the general population and to subclinical atherosclerosis in patients with rheumatoid arthritis and systemic lupus erythematosus.

Objectives: The main purpose of our study was to assess, for the first time, whether CEC is disrupted in patients with SS compared to controls. We also aimed to identify patients' characteristics that could explain such potential CEC disturbance.

Methods: Cross-sectional study that encompassed 188 individuals; 73 SS patients and 115 age- and sex-matched controls. CEC, using an in vitro assay, and lipoprotein serum concentrations were assessed in patients and controls. A multivariable analysis was performed to study the differences in CEC between patients and controls, and if SS-related data could explain CEC differences.

Results: CEC was downregulated in SS patients as compared to controls (beta coefficient $-6[95 \% \mathrm{Cl}-10--2] \%, p=0.002)$. This occurred independently of traditional cardiovascular risk factors, statin use or other variations in the lipid profile produced by the disease. Demographics and lipid profile were, in general, not related with CEC in both patients and controls. In this sense, only abdominal circumference showed a positive association with CEC in patients (beta coefficient 0.09 [95\% Cl $0.03-0.14], p=0.002$ ) but not in controls. Similarly, no traditional cardiovascular risk factors were related with CEC in both populations. Regarding lipid profile, no correlations were identified between the standard lipid profile molecules and CEC. Remarkably, the use of statins was not related to CEC in both patients and controls. Lastly, concerning SS related data, a negative association between $\mathrm{mRSS}$ and CEC was identified (beta coef. -1.08 [95\% Cl -2.03- -0.12$]$ $\%, p=0.028$ ).

Skin thickness through modified Rodnan (mRSS) was positively related to age and the presence of hypertension, but negatively associated with apolipoprotein $\mathrm{B}$, apo $\mathrm{B}: \mathrm{A} 1$ ratio, and $\mathrm{CEC}$ when univariate correlations were assessed (Table 4). When the relation of mRSS to these lipid-related molecules was adjusted for traditional CV risk factors, the statistical significance of mRSS with those molecules was maintained. Moreover, when the relation between mRSS and CEC was additionally adjusted for other lipid-related molecules, its significance was conserved (beta coef. $-1.35[95 \% \mathrm{Cl}-2.62--0.08]$ ) \% $\mathrm{p}=0.038$ )

Conclusion: CEC is downregulated in SS patients independently of other inflammation-related lipid profile modifications that occur in the disease. Skin thickness is independent and inversely associated with CEC in SS patients.

Disclosure of Interests: Iván Ferraz-Amaro Grant/research support from: Pfizer, Abbvie, Speakers bureau: Pfizer, Abbvie, MSD., delgado frias esmeralda Speakers bureau: Pfizer, Abbvie, MSD, Vanessa Hernández-Hernández Speakers bureau: Pfizer, Abbvie, MSD, Hiurma Sánchez-Pérez: None declared, Laura de Armas-Rillo: None declared, Estefania Armas González: None declared, Jose David Machado: None declared, Federico Díaz-González Grant/research support from: Abbvie, Pfizer, MSD, Speakers bureau: Abbvie, Pfizer, MSD

DOI: 10.1136/annrheumdis-2020-eular.620

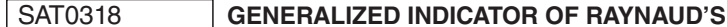 PHENOMENON EXPRESSION FOR EVALUATION OF CLINICAL EFFICACY OF PROSTANOID THERAPY}

I. Gaisin ${ }^{1}$, Z. Bagautdinova ${ }^{2}$, M. Glavatskikh ${ }^{3}$, N. Maximov ${ }^{1}$, R. Valeeva ${ }^{1}$, O. Desinova ${ }^{4}$, R. Shayakhmetova ${ }^{4} .{ }^{1}$ Izhevsk State Medical Academy, Izhevsk, Russian Federation; ${ }^{2}$ Clinical Diagnostic Centre of the Udmurt Republic, Izhevsk, Russian Federation; ${ }^{3}$ Udmurt State University, Izhevsk, Russian Federation; ${ }^{4}$ VA. Nasonova Research Institute of Rheumatology, Moscow, Russian Federation

Background: Raynaud's phenomenon (RP) secondary to rheumatic diseases (RD) can progress to irreversible tissue damage with digital ulceration, scarring and, rarely, gangrene requiring amputation ${ }^{1}$. Current medical treatments for RP are far from ideal: they are often either ineffective and/or poorly tolerated, thus a significant proportion of patients discontinue drug therapy ${ }^{2}$.

Objectives: To determine RP expression levels and to evaluate the long-term efficacy of iloprost and alprostadil in RP patients with RD.

Methods: Indicated therapy with intravenous iloprost $(n=10)$, alprostadil $(n=17)$ or their combinations $(n=13)$ was carried out for three years in patients with secondary RP in RD. Frequency of Raynaud's attacks, digital ulcers (DU) formation and pain intensity on visual analogue scale (VAS) were evaluated. A control group included 30 patients with RP in RD who did not receive prostanoid therapy. By factor analysis method a generalized index of $\mathrm{RP}$ expression was identified, on the basis of which levels of RP expression were determined.

Results: "RP expression" scale, revealed as an indicator of RP generalized manifestation, was an average value of two subscales: (1) consisted of 4 indices "DU", "digital pitting scars", "phalange amputation" and "frequency of Raynaud's attack", (2) included 3 indicators: "intensity of pain", "duration of illness", "whitening of fingers". Correlation of subscales showed their reliability $(r=0.294$, $p=0.053$ ). RP final expression (severity) was $1.51 \pm 0.86$. A low level of RP expression had values below 0.65 , a high level - over 2.37. At baseline, the high level of RP severity was defined in 16 (22.9\%) patients, medium - in 43 $(61.4 \%)$, low - in 11 (15.7\%).

RP treatment with iloprost was effective in the healing of DU in $100 \%$ of patients and led to decrease of RP expression generalized index from $2.25[1 ; 3]$ to 1.75 $[1 ; 2](p=0.012)$. Alprostadil therapy reduced pain intensity on VAS $(p<0.05)$ and numbness during Raynaud's attacks $(p<0.01)$ and decreased RP expression from $1[1 ; 2]$ to $1[0.5 ; 1.5](p=0.038)$. Patients on prostanoids combination had new DU and amputations; pain intensity reduced by $47 \%(p<0.05)$, RP expression generalized indicator did not change.

Conclusion: Based on RP clinical manifestations in RD patients, a generalized index of RP expression was identified and levels of RP severity were determined. Treatment with iloprost or alprostadil has significant effects on reducing the clinical manifestations of RP with a corresponding decrease in its severity. lloprost is indicated in patients with medium and high levels of RP expression index alprostadil - with medium and low index and non-effectiveness of calcium channel blockers. 
References:

[1] Hughes M, Herrick AL. Digital ulcers in systemic sclerosis. Rheumatology (Oxford) 2017;56 (1):14-25.

[2] Kowal-Bielecka O, Fransen J, Avouac $\mathrm{J}$ et al. Update of EULAR recommendation for the treatment of systemic sclerosis. Ann Rheum Dis 2017;76(8):1327-39.

Acknowledgments: Professor LP. Anan'eva

Disclosure of Interests: Ilshat Gaisin Speakers bureau: Boehringer Ingelheim, KRKA, Berlin-Chemie Menarini, Sanofi, Zukhra Bagautdinova: None declared, Marianna Glavatskikh: None declared, Nikolay Maximov Speakers bureau: Pfizer, KRKA, Rosa Valeeva: None declared, Oxana Desinova: None declared, Rushana Shayakhmetova: None declared

DOI: 10.1136/annrheumdis-2020-eular.2263

\begin{tabular}{|l|l}
\hline SAT0319 & SUBCLINICAL ATHEROSCLEROSIS IN INDIAN \\
& PATIENTS WITH SCLERODERMA - CLINICAL AND \\
& SEROLOGICAL ASSOCIATIONS
\end{tabular}

H. Gangadharan Nair ${ }^{1}$, M. K. Rai ${ }^{1}$, M. Singh ${ }^{2}$, A. Anuja ${ }^{1}$, K. Singh ${ }^{1}$, N. Mohindra ${ }^{3}$, N. Jain ${ }^{3}$, S. Kumar ${ }^{4}$, V. Agarwal ${ }^{1}$, D. Misra ${ }^{1} .{ }^{1}$ Sanjay Gandhi Postgraduate Institute of Medical Sciences, Department of Clinical Immunology and Rheumatology, Lucknow, India; ${ }^{2}$ Sanjay Gandhi Postgraduate Institute of Medical Sciences, Department of Nephrology, Lucknow, India; ${ }^{3}$ Sanjay Gandhi Postgraduate Institute of Medical Sciences, Department of Radiodiagnosis, Lucknow, India; ${ }^{4}$ Sanjay Gandhi Postgraduate Institute of Medical Sciences, Department of Cardiology, Lucknow, India

Background: Scleroderma has been associated with increased risk of cardiovascular events, however,studies on this from India are sparse.We evaluated clinical and serological factors associated with subclinical atherosclerosis in Indian patients with scleroderma, in a cross-sectional design.

Objectives: To compare carotid intima-medial thickness (CIMT, mean value of both carotids) as a measure of subclinical atherosclerosis (SCA) between patients with scleroderma $(n=61)$ fulfilling 2013 ACR/EULAR criteria, and healthy controls $(n=41)$.

- To compare clinical (body mass index - BMI, waist-hip ratio - WHR, fasting lipid profile) and serological factors (microparticles, endothelial microparticles, inflammatory cytokines associated with increased cardiovascular risk) between patients with scleroderma and healthy controls.

- To identify factors associated with SCA in scleroderma patients.

Methods: Subclinical atherosclerosis(SCA) was defined by presence of carotid plaques, or increased CIMT >2 standard deviations compared with Indian reference standards for age and sex. Total microparticles (TMP) were measured of plasma after ultracentrifugation as per previously described protocol using microbeads of $3 \mu \mathrm{m}$ size (TMP were of size 0.1-1 $\mu \mathrm{m}$ ); of these, microparticles positive for CD31 and CD142 were endothelial microparticles (EMP). Serum cytokines (IL-1, IL-6, TNF-a, IL-17) were measured by ELISA using manufacturer instructions. Linear regression was used to identify the determinants of CIMT in scleroderma. Binomial logistic regression was used to identify factors associated with subclinical athersclerosis in scleroderma.

\begin{tabular}{|c|c|c|c|}
\hline Variable & $\begin{array}{l}\text { Patients with sclero- } \\
\text { derma }(n=61)\end{array}$ & $\begin{array}{l}\text { Healthy controls } \\
\qquad(n=41)\end{array}$ & $\mathrm{p}$ value \\
\hline Age & $37.8 \pm 11.92$ & $35.37 \pm 6.69$ & 0.2375 \\
\hline Gender (M:F) & $11: 50$ & $6: 35$ & 0.6516 \\
\hline Diabetes/Hypertension/Tobacco use & $1 / 2 / 0$ & $0 / 0 / 0$ & NS \\
\hline Body mass index (kg/m2) & $20.11 \pm 3.82$ & $24.38 \pm 4.45$ & $<0.0001$ \\
\hline Waist-hip ratio & $0.86 \pm 0.11$ & $0.89 \pm 0.07$ & 0.1251 \\
\hline Total cholesterol (mg/dL) & $142.5 \pm 30.7$ & $147.3 \pm 39.5$ & 0.4948 \\
\hline Triglycerides (mg/dL) & $99.4 \pm 37$ & $121.4 \pm 46$ & 0.0087 \\
\hline HDL cholesterol (mg/dL) & $46.9 \pm 4.9$ & $46.1 \pm 4.2$ & 0.4029 \\
\hline LDL cholesterol (mg/dL) & $93.6 \pm 10.5$ & $93.3 \pm 7.5$ & 0.8520 \\
\hline VLDL cholesterol (mg/dL) & $19.9 \pm 7.4$ & $24.7 \pm 9.7$ & 0.0057 \\
\hline Carotid intima-medial thickness (mm) & $0.68 \pm 0.10$ & $0.53 \pm 0.03$ & $<0.0001$ \\
\hline Total microparticles (per $\pm \mathrm{L}$ ) & $12913 \pm 2493$ & $6272 \pm 1533$ & $<0.0001$ \\
\hline Endothelial microparticles (per $\pm \mathrm{L}$ ) & $2623 \pm 1032$ & $829 \pm 439.5$ & $<0.0001$ \\
\hline Serum IL-1 $\pm(\mathrm{pg} / \mathrm{mL})$ & $38.19 \pm 13.46$ & $31.38 \pm 18.29$ & 0.0326 \\
\hline IL-6 (pg/mL) & $176.6 \pm 85.74$ & $128.9 \pm 53.61$ & 0.0020 \\
\hline IL-17 (pg/mL) & $56.3 \pm 20.45$ & $53.89 \pm 20.51$ & 0.5611 \\
\hline $\mathrm{TNF}_{ \pm}(\mathrm{pg} / \mathrm{mL})$ & $49.65 \pm 26.71$ & $42.09 \pm 30.41$ & 0.1879 \\
\hline
\end{tabular}

Results: Despite lower BMI, triglycerides and VLDL cholesterol, CIMT was significantly higher in patients with scleroderma. Patients with scleroderma had significantly higher total microparticles and endothelial microparticles in plasma, and serum IL-1 \pm and IL-6 (Table 1). On multivariable regression, age was the only significant determinant of CIMT. 28 (45.9\%) patients had SCA; $13(21.3 \%)$ had carotid plaques. Patients with SCA had higher proportion of males (9/28 in those with SCA vs $2 / 33$ in those without SCA). Binomial logistic regression did not identify any other significant predictors of SCA.

Table 1. Comparison between patients with scleroderma and healthy controls

\begin{tabular}{llll}
\hline Serum IL-1 $\pm(\mathrm{pg} / \mathrm{mL})$ & $38.19 \pm 13.46$ & $31.38 \pm 18.29$ & 0.0326 \\
$\mathrm{IL}-6(\mathrm{pg} / \mathrm{mL})$ & $176.6 \pm 85.74$ & $128.9 \pm 53.61$ & 0.0020 \\
$\mathrm{IL}-17(\mathrm{pg} / \mathrm{mL})$ & $56.3 \pm 20.45$ & $53.89 \pm 20.51$ & 0.5611 \\
$\mathrm{TNF} \pm(\mathrm{pg} / \mathrm{mL})$ & $49.65 \pm 26.71$ & $42.09 \pm 30.41$ & 0.1879 \\
\hline
\end{tabular}

Conclusion: Patients with scleroderma had significant burden of subclinical atherosclerosis, which could not be explained by traditional or novel cardiovascular risk factors.

\section{References:}

[1]Psarras A, Soulaidopoulos S, Garyfallos A, Kitas G, Dimitroulas T. A critical view on cardiovascular risk in systemic sclerosis. Rheumatol Int. 2017 Jan; 37(1):85-95.

[2]Jung C, Drummer K, Oelzner P, Figulla HR, Boettcher J, Franz M, et al. The association between endothelial microparticles and inflammation in patients with systemic sclerosis and Raynaud's phenomenon as detected by functional imaging. Clin HemorheolMicrocirc. 2015; 61(3):549-557.

Acknowledgments: Supported by IRA(Indian Rheumatology Association) Research Grant to DP Misra.

Disclosure of Interests: None declared

DOI: 10.1136/annrheumdis-2020-eular.296

\section{SAT0320 \\ BONE MINERAL DENSITY AND FRACTURE RISK IN A COHORT OF PORTUGUESE SYSTEMIC SCLEROSIS PATIENTS}

S. Garcia ${ }^{1}$, B. M. Fernandes ${ }^{1}$, S. Ganhão ${ }^{1}$, M. Rato ${ }^{1}$, F. Pinheiro ${ }^{1}$, G. Terroso ${ }^{1}$, M. Bernardes ${ }^{1}$, L. Costa ${ }^{1} .{ }^{1}$ Centro Hospitalar Universitário São João, Porto, Portugal

Background: Although poorly understood, patients with Systemic Sclerosis (SSc) seem to have higher prevalence of low bone mineral density (BMD) and an increased spine fracture risk.

Objectives: We aim to determine, by conventional densitometry (DXA) and using the fracture risk assessment tool (FRAX), the prevalence of low BMD and the fracture risk, respectively, in our SSc cohort and its potential determinants. Methods: Observational transversal study was performed including consecutive patients with the diagnosis of SSc. We collected data regarding demographics BMD (lumbar spine and femoral neck) and occurrence of fracture. Ten-year risk of osteoporotic fracture was estimated using FRAX v4.1 with the Portuguese population reference. Statistical analysis was performed using SPSS 23.0; $\mathrm{p}<0.01$ was considered statistically significant.

Results: Median age of patients $(n=97)$ was 62 years old [56, 70], 88.7\% females $(n=86)$. Seventy-eight patients $(80.4 \%)$ had limited cutaneous form, 5 (5.2\%) presented a diffuse cutaneous form and $13(13.4 \%)$ an overlap syndrome. Regarding clinical features: digital ulcers in 30 patients $(30.9 \%)$, interstitial lung disease (ILD) in 16 (6.5\%), gastrointestinal involvement in $16(16.5 \%)$, miositis in $4(4.1 \%)$ and pulmonary arterial hypertension in $3(3.1 \%)$. Anti-topoisomerase I antibody (anti-Scl70) positivity was present in 15 patients (15.5\%) and anti-centromere antibody (ACA) positivity in 63 (64.9\%). Nine patients $(9.3 \%)$ were smokers and $6(6.2 \%)$ reported an alcohol consumption of 3 or more units/ day. Median body mass index (BMI) was $25.4 \mathrm{Kg} / \mathrm{m}^{2}$ [21.4, 29.1], with 5 patients $(5.2 \%)$ being underweight. Vitamin D insufficiency was reported in 19 patients $(19.6 \%)$. Twenty-one patients $(21.6 \%)$ have been exposed to oral glucocorticoids (GCT) for more than 3 months at a dose of $5 \mathrm{mg}$ daily or more. Eleven patients (11.3\%) had previous low impact fractures: 10 of which were vertebral and 1 wrist fracture. Regarding the prescribed anti-osteoporotic treatment (AOP), we found: alendronate $(n=7,7.2 \%)$, zoledronic acid $(n=7,7.2 \%)$, denosumab $(n=2,2.1 \%)$ and teriparatide $(n=1,1 \%)$.

Low BMD was present in 45 patients (46.4\%); median femoral neck BMD (FN-BMD) was $0.827[0.709,0.893]$. Ten year probability of fracture (\%) was: median risk for major fracture was $5.1[3.5,9.7]$ and $3.8[2.5,8]$, with and without FN-BMD, respectively; for hip fracture the estimated risk was $1.2[0.6,3.1]$ and $1.0[0.4,2.5]$, with and without FN-BMD, respectively. According to FRAX thresholds for the Portuguese population, 25 patients $(25.8 \%)$ met criteria to start AOP treatment. Among them, only 10 patients (40\%) started it, as the agreement between the indication to treat by FRAX and the onset of treatment was weak ( $k=$ 0.338). A strong agreement was found between FRAX risk threshold with DXA and World Health Organization (WHO) threshold for starting AOP $(k=0.814)$ and no agreement was found between FRAX risk without DXA and WHO threshold. FN-BMD presented a weak correlation with BMI $(r=0.393)$, a moderate inverse correlation with major fracture risk with and without FN-BMD $(r=-0.704$, 